\title{
Sachkapitalrenditen im historischen Vergleich - Deutschland im Abwärtstrend?
}

Die Finanzkrise, die 2007 in den USA ausbrach, weckte wegen ihres historischen Ausmaßes und ihrer globalen Reichweite Erinnerungen an die Weltwirtschaftskrise 1929. Die Krise warf grundsätzliche Fragen nach Stabilität und langfristiger Tendenz der Weltwirtschaft auf. Vor diesem Hintergrund wird im Folgenden eine wichtige Frage kapitalistisch verfasster Ökonomien am Beispiel der Bundesrepublik Deutschland ab 1970 empirisch untersucht: Wie entwickelte sich langfristig die Rendite des Sachkapitals (Rentabilität) oder, in der Sprache der klassischen politischen Ökonomie, die „Profitrate“? ${ }^{1}$

THOMAS WEIß

\section{Einleitung}

Die Große Rezession ab 2007 erfolgte vor dem Hintergrund sich seit Langem weltweit abschwächender wirtschaftlicher Wachstumsraten und eines Rückgangs der Investitionsquoten. ${ }^{2}$ Thesen zur "Stagnation“ des Kapitalismus haben Konjunktur (z. B. Summers 2013; Krugman 2013). Dabei wird auch der „langfristigen marxistischen Perspektive“, dem „tendenzielle(n) Fall der Profitrate“, wieder „wachsende Aktualität“" zugesprochen (Klär 2013, S. 227).

Als Ursachen für nachlassendes Wachstum und Investitionsschwäche nennen alte ${ }^{3}$ und neue Stagnationstheoretiker eine Verlangsamung des technischen Fortschritts, die nachlassende Bereitschaft zu riskantem unternehmerischen Handeln oder politische Reibungen (vgl. Nachtwey 2015, S. 7). Schon klassische Ökonomen wie Robert Malthus sahen in demografischen Entwicklungen und der Erschöpfung natürlicher Ressourcen Wachstumsschranken. Nach David Ricardo erzwingt eine wachsende Bevölkerung die Bewirtschaftung zusätzlicher, immer weniger fruchtbarer landwirtschaftlicher Böden mit immer größerem Arbeitsaufwand für die Nahrungsmittelproduktion. Nahrungsmittelpreise richten sich nach dem hohen Arbeitsaufwand dieser „marginalen“ Böden. Grundeigentümer mit Böden, deren Fruchtbarkeit höher als die marginale ist und daher niedrigere Produktionskosten haben, beziehen eine immer größer werdende Differential-Bo- denrente. Mit den Preisen müssen die Löhne steigen, sollen die Arbeiter sich weiterhin ernähren können. Dies senkt die „Profitrate“ in der Industrie.

Karl Marx führte Stagnation weniger auf äußere Schranken als auf innere „logische Widersprüche des kapitalistischen Systems“ zurück (vgl. Piketty 2014, S. 23). Lohnarbeit, so Marx, wird wegrationalisiert und durch fixes Kapital in Form von Maschinen ersetzt. Ziel des einzelnen Kapitalisten

1 Ich bedanke mich für jeweils wertvolle Auskünfte bzw. Hinweise bei Mitarbeitern und Mitarbeiterinnen des Statistischen Bundesamtes und der Deutschen Bundesbank sowie bei zwei anonymen Gutachtern der WSI-Mitteilungen.

2 Vgl. Internationaler Währungsfonds (IWF) (2005, S. 91ff.) Eine grafische Darstellung von IWF-Daten zu Wachstumsraten und Investitionsquoten, jeweils für die Welt und die OECD, findet sich mit Quellenangabe bei Wikipedia unter: https://commons.wikimedia.org/wiki/File:WeltBIPWorld groupOECD.PNG und https://commons.wikimedia.org/ wiki/File:IQuoteWeltEngl.PNG. Die in den USA langfristig fallende Kapazitätsauslastung in der Industrie wird von der Federal Reserve Bank hier abgebildet: http://www.federalreserve.gov/releases/g17/current/ipg1.gif. Zur Bundesrepublik Deutschland finden sich Daten ab der Nachkriegszeit in Statistisches Bundesamt (2014a).

3 Der Keynes-Schüler Alvin Hansen prägte in den 1930er Jahren den Begriff der "säkularen Stagnation“ (vgl. Deutsche Bundesbank 2014a). 
ist es, die Einnahmen im Vergleich zu den Lohnkosten zu steigern. Mit Hilfe von Maschinen wird die Arbeitsproduktivität erhöht. Im Vergleich zum Wert des fortlaufend akkumulierten fixen Kapitals fällt der Einsatz an Arbeitskräften zurück. Die Wertschöpfung bemisst sich dabei gemäß klassischer Arbeitswertlehre nach der „notwendigen Arbeitszeit", nicht nach der Menge der Produkte. ${ }^{4}$ Rationalisierungsinvestitionen vermindern also das Beschäftigungswachstum. Mit zurückbleibendem Arbeitseinsatz halten die als Einkommen verteilbare Wertschöpfung und damit auch der Profit als Teil der Wertschöpfung nicht mit der Kapitalakkumulation Schritt (vgl. z. B. Krüger 2012, S. 251ff.). Die Profitrate oder Rentabilität fällt. Diesen Zusammenhang bezeichnet Piketty (2014, S. 23, 301) als „Prinzip der unbegrenzten Akkumulation“. 5

Im Gegensatz zu Ricardo rechnete Marx mit einer sinkenden Lohnquote. Der den Kapitalisten zukommende „Mehrwert" steigt als Teil der Wertschöpfung. Aber der Einsatz an fixem Kapital oder die Kapitalintensität (Kapitalstock je Beschäftigten) steigt so stark, dass die Kapitalproduktivität (Verhältnis Wertschöpfung zu Kapitalstock) und „tendenziell“ die Rentabilität sinken. ${ }^{6}$ Mit dem Fall der Profitrate erlahmt der „Stachel der kapitalistischen Produktion“. In dieser „marxistischen apokalyptischen Vision“ (Piketty 2014, S. 13) verlangsamt sich so die „Bildung neuer selbstständiger Kapitale“. Der kapitalistische Produktionsprozess ist bedroht durch „Überproduktion, Spekulation, Krisen... “ (Marx/Engels 1987, S. 252) oder in Pikettys (2014, S. 24) Worten: Der Motor der Akkumulation wird abgewürgt, und womöglich „zerfleischen“ sich die Kapitalisten gegenseitig.

Streng genommen muss das Wegrationalisieren von Arbeitsplätzen nicht unmittelbar Produktion und Investition schwächen. So stellt Lindlar (1997, S. 275) für die 1950er bis 1980er Jahre für Großbritannien und die USA trotz gleichzeitig sinkender Sachkapitalrenditen eine steigende Brutto-Investitionsquote fest. Vieles spricht aber dafür, dass langfristig das wirtschaftliche Wachstum ,verebben“ muss (Arnold 1997, S. 5; vgl. auch Mattfeldt 2006, S. 11), etwa weil mit dem Fall der Profitrate die Kapitalkonzentration voranschreitet (vgl. Callinicos 2014, S. 276; vgl. Marx/Engels 1987, S. 269).

John Maynard Keynes schließlich begründete eine eigene Stagnationsschule. Ihm zufolge bleibt der Konsum, etwa wegen Sättigung der Bedürfnisse, hinter den Produktionsmöglichkeiten zurück. Wird zum Ausgleich mehr investiert, macht sich wieder „das Prinzip der unbegrenzten Akkumulation“ (Piketty 2014, S. 23, 301) bemerkbar. Auch Investitionen müssen letztlich durch die Konsumnachfrage begründet werden. Wächst die Kapitalakkumulation über die durch die Bedürfnisse bestimmte Nachfrage hinaus an, sinkt die „Grenzleistungsfähigkeit des Kapitals“ (Rentabilität, Profitrate). Es droht zugespitzt die „Euthanasie des Rentiers“, also ein Niedrigzinsregime. Keynes (2009, S. 318f.) schlug sogar eine „ziemlich umfassende gesamtwirtschaftliche Steuerung der Investitionen" vor, um stagnative Tendenzen zu bannen (zu Parallelen zu Marx vgl. Krüger 2012, S. 288).
Vor diesem theoretischen Hintergrund soll im Folgenden empirisch untersucht werden, wie sich die Rentabilität oder „Profitrate“ der Unternehmen in der Bundesrepublik Deutschland seit 1970 entwickelt hat. ${ }^{7}$ Besonders untersucht wird die Kapitalproduktivität. Fiel die Wertschöpfung, aus der sich die Einkommen von Arbeit und Kapital speisen, im Verhältnis zum Einsatz an Kapital zurück? Ist dies der Fall, kann eine Verminderung der Lohnquote, wie sie in der Bundesrepublik etwa seit den 1980er Jahren zu beobachten ist, nicht nachhaltig die Rentabilität stützen. Abschließend werden einige wirtschaftspolitische Schlussfolgerungen gezogen.

\section{Begriffe und Daten}

\subsection{Konzepte und statistische Umsetzung}

In kapitalistischen Ökonomien wird Kapital investiert, um einen Gewinn zu erzielen. Der Gewinn im Verhältnis zum investierten Kapital ist die Rentabilität. Der Gewinn eines Jahres wird auf den Kapitalbestand zu Jahresbeginn bezogen. ${ }^{8}$ Als eine Art „Sensitivitätsanalyse“ zur Prüfung der „Robustheit“ der Ergebnisse werden hier zwei sich konzeptionell unterscheidende Datensätze des Statistischen Bundesamts (StBA) und der Deutschen Bundesbank verwendet.

4 Dagegen nimmt in neoklassischen Produktionsfunktionen die Produktion je Arbeitskraft immer weniger zu, wenn der Kapitaleinsatz je Arbeitskraft steigt. Diese sinkende marginale Kapitalproduktivität führt dann zu einem Fall der Profitrate. Technischer Fortschritt, der die Produktion je Arbeitseinsatz erhöht, verhindert dann den Profitratenfall (so z. B. kritisch gegen Marx Piketty 2014, S. 24, 302). Piketty deutet neoklassisch die Profitrate stofflich, also als einen in Produktionsmengen gemessenen Ertrag des Kapitals. Zu einer aktuellen empirischen Studie zur Arbeitswertlehre vgl. Fröhlich (2010).

5 "Trop de capital tue le capital“ - „Zuviel Kapital tötet das Kapital“ (Piketty 2014, S. 285).

6 „Die Profitrate fällt, nicht weil der Arbeiter weniger exploitiert wird, sondern weil im Verhältnis zum angewandten Kapital überhaupt weniger Arbeit angewandt wird" (Marx/ Engels 1987, S. 256). Zu Pro und Contra dieser Argumentation vgl. z. B. Heinrich (2004, S. 148ff.)

7 Frühere Untersuchungen zu dieser Frage sind z. B. Priewe (1988); Bontrup (2000); Mattfeldt (2006).

8 Vgl. Bitz et al. (2011, S. 640). Im Folgenden wird allerdings jeweils der für das Jahresende ausgewiesene Kapitalbestand verwendet, was die Ergebnisse "fast nicht" verändert (Räth et al. 2011, S. 853). 


\subsubsection{Volkswirtschaftliche Gesamtrechnungen}

Der Gewinn ist die Differenz aus Einnahmen und Ausgaben eines Unternehmens. In den Volkswirtschaftlichen Gesamtrechnungen (VGR) des StBA kann dies dargestellt werden als Produktionswert oder Umsatz aller Unternehmen abzüglich der Vorleistungen, also der von anderen Unternehmen bezogenen und im Berichtsjahr verbrauchten Waren. ${ }^{9}$ Diese „Bruttowertschöpfung“ setzt sich aus dem Einkommen aus Arbeit (Arbeitnehmerentgelt) und dem Einkommen aus Kapital zusammen. Nach Abzug des Kostenfaktors „Abschreibungen“ für die Abnutzung der Sachanlagen verbleibt die eigentliche Gewinngröße. Dies ist der „Nettobetriebsüberschuss“ mit dem "Selbstständigeneinkommen“. Dieser Gewinn enthält auch den kalkulatorischen Unternehmerlohn und das Entgelt für das eingesetzte eigene und fremde Sach- und Geldkapital („Forderungsvermögen“) vor Abzug der Steuern.

Die Rentabilität bzw. die Kapitalproduktivität ergibt sich, indem dieser Gewinn bzw. die Bruttowertschöpfung insgesamt auf das Bruttoanlagevermögen zu Wiederbeschaffungspreisen bezogen wird. Das ist der Geldbetrag, der gezahlt werden müsste, wenn die Anlagen zum Berichtszeitpunkt neu angeschafft würden (Räth et al. 2011, S. 852). Anlagegüter werden nach diesem „Bruttokonzept" nicht allmählich abgeschrieben, sondern scheiden erst zum Ende ihrer Lebensdauer aus dem Anlagevermögen aus. Dieses umfasst die produzierten Vermögensgüter, also Bauten, Ausrüstungen, immaterielle Anlagegüter, Nutztiere und Nutzpflanzen. Alle Größen werden zu laufenden Preisen bewertet. Bei den Abschreibungen und beim Anlagevermögen sind dies die jeweiligen Wiederbeschaffungspreise (vgl. Bitz et al. 2011, S. 76; Funke 1987, S. 190f.). ${ }^{10}$

\subsubsection{Jahresabschlüsse}

Zur Berechnung der "Gesamtrentabilität“ (Bitz et al. 2011, S. 639) ergibt sich bei den Jahresabschlüssen, wie sie die Bundesbank veröffentlicht, die Gewinngröße „Jahresergeb- nis vor Gewinnsteuern“ als Differenz von Ertrag und Aufwand. Nach Abzug der Gewinnsteuern verbleibt das ,Jahresergebnis“ (den Eigenmitteln zurechenbarer Gewinn). Dazu kommen die Zinsaufwendungen (den Fremdmitteln zurechenbarer Gewinn).

Die Erträge sind dabei die „Leistung“, also Umsatz plus Bestandsveränderungen an Erzeugnissen. Dies entspricht dem Produktionswert der VGR. Dazu kommen - im Unterschied zu den VGR - als Ertragsgrößen noch Zins- und übrige Erträge, die auch Erträge aus Beteiligungen enthalten. Der vom Ertrag abzuziehende Aufwand besteht aus Materialaufwand, dem in den VGR die Vorleistungen entsprechen, Personalaufwand (entspricht dem Arbeitsentgelt), Abschreibungen und Zinsaufwendungen, schließlich auch Betriebssteuern und übrige Aufwendungen (Deutsche Bundesbank 2013, S. 10).

Als Kapitaleinsatz wird bei der Bilanzkennzahl „Gesamtrentabilität" die Bilanzsumme der Unternehmen verwendet. Neben Rechnungsabgrenzungsposten umfasst diese auf der Aktivseite nicht nur das Sachvermögen, sondern auch das „Forderungsvermögen“. Das Sachvermögen besteht aus „immateriellen Vermögensgegenständen“, darunter „Geschäftsoder Firmenwert“, und „Sachanlagen“, das sind „Grundstücke und Gebäude“ und „Vorräte“. Das Forderungsvermögen setzt sich aus „Kasse und Bankguthaben“, „Forderungen“, „Wertpapieren“ und „Beteiligungen“ zusammen. Auf der Passivseite werden „Eigenmittel“ („Eigenkapital“ und „Rücklagen“) und „Fremdmittel“ (kurz- und langfristige Verbindlichkeiten sowie „Rückstellungen“) ausgewiesen. Der Kapitalbegriff dieser Bilanzsumme unterscheidet sich von dem der VGR, weil nicht nur das produzierte Anlagevermögen („fixes Kapital“), sondern auch der Wert von Grundstücken sowie das Forderungsvermögen dazu zählt. ${ }^{11}$

Abschreibungen sowie die Bestandteile der Bilanz sind nach geltenden Rechnungslegungsstandards der betrieblichen Buchhaltung bewertet, also in der Regel nach Anschaffungspreisen, nicht nach Wiederbeschaffungspreisen (vgl. Bitz et al. 2011, S. 156; Schmalwasser/Schidlowski 2006, S. 1110; Priewe 1988, S. 237). ${ }^{12}$ Der Wert der Sachanlagen
9 Dies ist eine vereinfachte Darstellung, vgl. zu den Einzelheiten z. B. Arbeitskreis „Volkswirtschaftliche Gesamtrechnungen der Länder" (2014), siehe insbesondere das "Schaubild".

10 In der während der 1980er Jahre zwischen dem Deutschen Institut für Wirtschaftsforschung (DIW) und dem Rheinisch-Wesfälischen Institut für Wirtschaftsforschung (RWI) ausgetragenen Kontroverse bedeutete Bewertung der Anlagen zu Anschaffungskosten eine eher günstige, zu Wiederbeschaffungspreisen eine ungünstige Rentabilitätsentwicklung, jeweils inflationsbedingt (vgl. Funke 1987, S. 191; Priewe 1988, S. 243). Im heutigen disinflationären Umfeld ist es eher umgekehrt (Kliman 2012, S. 102ff.). Die "reale“ Kapitalproduktivität ist preisbereinigte Bruttowertschöpfung oder BIP im Verhältnis zum Anlagevermögen in konstanten Preisen. Ähnlich ist die Arbeitsproduktivität als preisbereinigtes BIP je Erwerbstätigen oder je Erwerbs- tätigenstunde definiert. Diese Größe wird in diesem Beitrag nicht betrachtet. Ihr Verlauf unterscheidet sich von der hier dargestellten Kapitalproduktivität, wenn die Preisindizes für Wertschöpfung und Anlagevermögen, also für den Zähler und den Nenner der Größe, sich im Verlauf stärker unterscheiden. Eigene Berechnungen ergaben für die 1970 er bis 1980er Jahre einen etwas schwächeren Rückgang der „realen“ Kapitalproduktivität als bei der hier ermittelten.

11 Seit 1991 liegt eine analoge Größe der VGR vor, die von StBA und Deutsche Bundesbank gemeinsam veröffentlicht wird (Statistisches Bundesamt/Deutsche Bundesbank 2013).

12 Das StBA bedient das Anschaffungspreiskonzept seit den 1990er Jahren nicht mehr und es ist im Europäischen System Volkswirtschaftlicher Gesamtrechnungen (ESVG) nicht vorgesehen. 
wird nach dem sogenannten Nettoprinzip als Nettoanlagevermögen ausgewiesen. Er wird also um die Abschreibungen vermindert. Erhöht um die für die Ersatzinvestitionen als Geldvermögen akkumulierten Abschreibungen ist man wieder beim Bruttoanlagevermögen. Insofern ist es hier sinnvoll, neben dem nach dem Nettoprinzip ausgewiesenen Sachanlagevermögen auch das Forderungsvermögen, das Rückstellungen für Reinvestitionen umfasst, als Bezugsgröße für die Rentabilität mit zu berücksichtigen.

\subsection{Datenmaterial}

Die Daten des StBA umfassen die gesamte deutsche Volkswirtschaft. Zwischen 1970 und 1991 beziehen sie sich auf das alte und seit 1991 auf das neue Gebiet der Bundesrepublik Deutschland. Die Zahlen seit 1991 entsprechen der VGR-Generalrevision 2014. Nach dieser zählen neuerdings u. a. Ausgaben für Forschung und Entwicklung (F\&E) als Investitionen (sonstige Anlagen) und nicht mehr, wie bis 1991, als in derselben Periode verbrauchte Vorleistungen (Statistisches Bundesamt 2014b). F\&E sind jetzt also Teil des Bruttoinlandsprodukts und des Kapitalbestandes. Sie werden auch über die Lebensdauer hinweg abgeschrieben.

Für die Jahresabschlüsse liegen drei Zeitreihen der Bundesbank vor, 1971 bis 1998 für Westdeutschland und 1997 bis 2009 sowie 2006 bis 2013 für Deutschland insgesamt. Ein größerer Strukturbruch zeigt sich zwischen der Zeitreihe 1971 bis 1998 und den beiden anderen Zeitreihen. Die erste Zeitreihe beruht auf Bilanzen und Erfolgsrechnungen, die dem deutschen Zentralbanksystem aufgrund des Rediskontgeschäfts mit Handelswechseln eingereicht wurden. Die neueren gesamtdeutschen Zahlen ab 1997 sind mithilfe eines Datenpools hochgerechnet, der gemeinsam von der Bundesbank und einigen anderen Finanzinstituten geführt wird (Deutsche Bundesbank 2005, S. 34). Zwei Drittel der Abschlüsse sind Steuerbilanzen (Deutsche Bundesbank 2013, S. $5 \mathrm{ff}.)^{13}$

\section{Ergebnisse}

\subsection{Kapitalproduktivität}

Die Entwicklung der Kapitalproduktivität als Obergrenze für die Rentabilität wird anhand der Daten des StBA in Abbildung 1 wiedergegeben. Vor allem im produzierenden Gewerbe (im Folgenden „Industrie“) erhöhte sich durch die Wiedervereinigung die Wertschöpfung, mehr noch der Kapitalbestand. Im Ergebnis liegt in der Industrie die Kapitalproduktivität ab 1991 deutlich niedriger als noch bis 1991 in der alten Bundesrepublik. Die konzeptionellen Änderungen der VGR-Generalrevision 2014 führen allerdings
ABB. 1

\section{Kapitalproduktivität (Bruttowertschöpfung im Verhältnis zu Bruttoanlage- vermögen zu Wiederbeschaffungspreisen)*}

Angaben in Prozent

$$
\begin{array}{lll}
\text { - Verarbeitendes Gewerbe } & \text { - Produzierendes Gewerbe } & \text { "...' K\&V }{ }^{2} \\
\text { - Unternehmen }{ }^{3} \text { ohne G\&W4 } & \cdots \text { Wirtschaft ohne G\&W } & \text { - Dienste ohne G\&W6 u. K\&V } \\
\text { - Alle Wirtschaftsbereiche } & - \text { G\&W }^{8}
\end{array}
$$

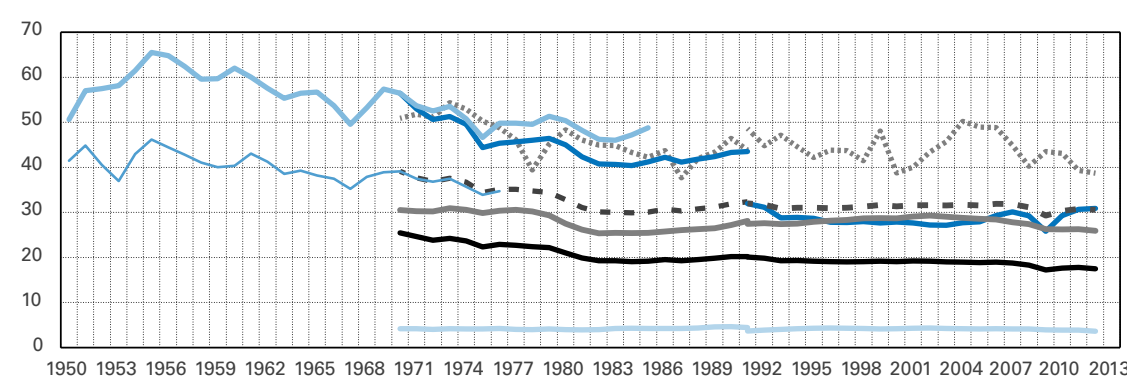

*bis 1991 altes, ab 1991 neues Bundesgebiet, bis 1991 vor, ab 1991 nach VGR-Revision.

1) konst. Preise, nach Priewe (1988). 2), 7) Kredit- und Versicherungsgewerbe. 3) Nettoanl.verm., nach Ipsen (1983) sowie Priewe (1988). 4), 5), 6), 8) Grundstücks- und Wohnungswesen.

Quelle: Statistisches Bundesamt; Berechnungen des Autors.

zu einer etwas höheren Kapitalproduktivität. Diese gegenläufigen Wirkungen bewirken, dass einige Reihen 1991 scheinbar bruchlos ineinander übergehen.

Eine starke Verzerrung ergibt sich durch das "Grundstücks- und Wohnungswesen“ (G\&W, „Immobilien-Bereich“). Es vereinigte $197039 \%$ und 2012 (nach Revision) $49 \%$ der Anlagen aller Wirtschaftsbereiche auf sich. Bei der Bruttowertschöpfung und dem Arbeitnehmerentgelt lauten die Werte dagegen jeweils 6 und $10 \%$ bzw. 0,3 und 0,8\%. Ausgerechnet in der gemessen am Kapitalbestand mit Abstand größten Branche spielt Arbeit kaum eine Rolle. Es herrscht „Vollautomatisierung“. Dabei sind, wie erwähnt, beim StBA im Unterschied zu den Zahlen der Bundesbank Grund und Boden als nicht produzierte Vermögensgüter nicht in der Anlagevermögensrechnung der VGR enthalten, sondern nur der Wert der Gebäude. Dass dieser Immobilienbereich an Bedeutung gewann, dämpfte die Entwicklung des gesamtwirtschaftlichen Durchschnitts der Kapitalproduktivität. $^{14}$

13 Die Zeitreihen unterscheiden sich bezüglich der jeweils erfassten Wirtschaftszweige, im Wesentlichen Produzierendes Gewerbe und Untergruppen des Dienstleistungsbereichs, vgl. Deutsche Bundesbank (1999, S. 8f.; 2009, S. 7, S. 9; 2013, S. 7f.; 2014b). Über grenzüberschreitende Vorgänge etwa wegen Steuervermeidung können die Daten verzerrt sein (vgl. Piketty 2014, S. 258, Fn. 1).

14 Zur Bedeutung von Grundeigentum und der darauf beruhenden Grundrente auf die kapitalistische Entwicklung vgl. Bischoff et al. (1993, S. 161ff.). 
ABB. 2

\section{Rentabilität (Nettobetriebsüberschuss im Verhältnis zu Bruttoanlage- vermögen zu Wiederbeschaffungspreisen)*}

Angaben in Prozent

$$
\begin{aligned}
& \text { - Verarbeitendes Gewerbe }{ }^{1} \quad \text { - Produzierendes Gewerbe '....' K\&V² } \\
& \text { — Unternehmen }{ }^{3} \text { ohne G\&W4 } \quad \cdots \text { Wirtschaft ohne G\&W5 } \quad \text { - Dienste ohne G\&W6 }{ }^{5} \text {. K\&V } \\
& \text { - Alle Wirtschaftsbereiche - G\&W }
\end{aligned}
$$

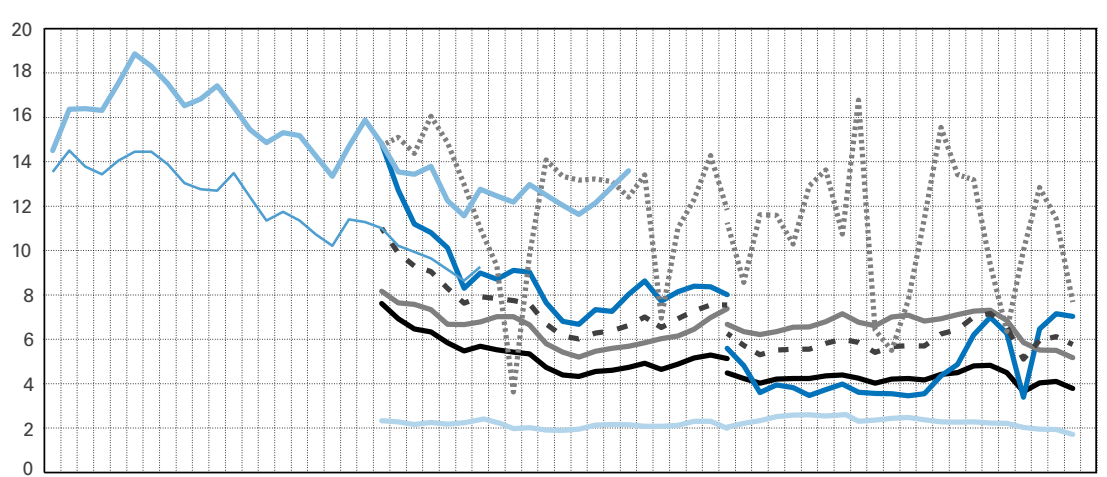

1950195319561959196219651968197119741977198019831986198919921995199820012004200720102013

*bis 1991 altes, ab 1991 neues Bundesgebiet, bis 1991 vor, ab 1991 nach VGR-Revision.

1) konst. Preise, nach Priewe (1988). 2), 7) Kredit- und Versicherungsgewerbe. 3) Nettoanl.verm., nach Ipsen/Priewe (1988). 4), 5), 6), 8) Grundstücks- und Wohnungswesen.

Quelle: Statistisches Bundesamt; Berechnungen des Autors.

ABB. 3

\section{Bilanzkennzahlen nichtfinanzieller Unternehmen}

verschiedene Zeitreihen 1971 - 1998, 1997 - 2009, 2006 - 2013

Angaben in Prozent der Bilanzsumme

- Gesamtrentabilität*

- Zinsaufwendungen

---- „strukturbruchbereinigt"

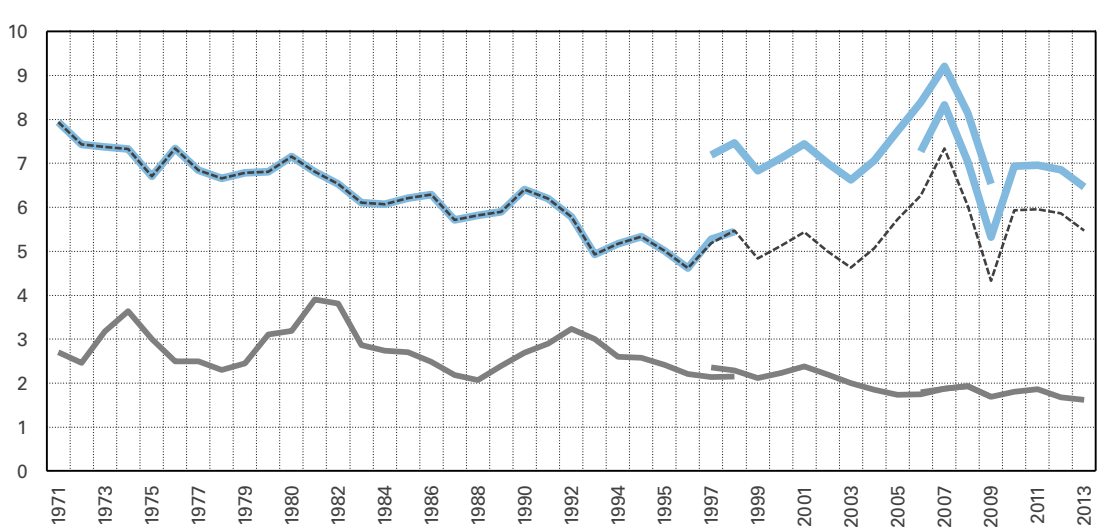

*Zinsaufwendungen zzgl. Jahresergebnis, drei Zeitreihen für 1971-1998, 1997-2009 und 2006-2013.

Quelle: Zahlen zu Jahresabschlüssen deutscher nichtfinanzieller Unternehmen der Deutschen Bundesbank; Berechnungen des Autors.
Insgesamt lassen sich zwei Zeiträume unterscheiden, vor und nach 1982. Dieses Jahr markiert gewissermaßen den „Paradigmenwechsel“ zwischen Keynesianismus und Neoliberalismus. ${ }^{15}$ Bis 1982 ging die Kapitalproduktivität kräftig zurück, die Entwicklung danach lässt sich auch als Seitwärtsbewegung verstehen - mit schwach sinkender Tendenz. Auffallend sind die „blasenförmigen“ Gipfel vor dem Krisentief 2009 - zuerst 2004 im Kredit- und Versicherungswesen (im Folgenden „Finanzsektor“), dann 2007 in der Industrie. Bemerkenswerterweise führte dieser Vorkrisenboom zu keinen Überhitzungserscheinungen (vgl. Klär 2014, S. 14).

\subsection{Rentabilität}

Eine veränderte Aufteilung der Arbeits- und Kapitaleinkommen kann die Rentabilität nur im Rahmen der Kapitalproduktivität verändern (Abbildung 2). Die seit etwa 1980 sinkende Lohnquote (für andere Länder siehe Piketty 2014, S. 294) hat in diesem Rahmen die Rentabilität gestützt. Im Finanzsektor schwankt diese stark und sinkt im Trend, unterbrochen von Einbrüchen wie 1987 (weltweiter Börsencrash), 2000/2001 (Dot.com-Krise) oder 2008 (weltweite Finanzkrise).

Schon wegen der genannten statistisch-methodischen Unterschiede, die sich allerdings in ihrer Wirkung auf die Rentabilität teilweise ausgleichen, ergibt sich aus den Daten der Bundesbank ein etwas anderes Bild. In Abbildung 3 sind die Zinsaufwendungen (die Gewinngröße für das eingesetzte Fremdkapital) und die Gesamtrentabilität als „Zinsaufwendungen“ zuzüglich „Jahresergebnis“ im Verhältnis zur Bilanzsumme abgebildet. (Das Jahresergebnis enthält die den Eigenmitteln zukommenden Gewinne). Die Rentabilität liegt ab 1997 wegen des Strukturbruchs höher als in der vorausgehenden Zeitreihe. ${ }^{16}$

Auch nach den Zahlen der Bundesbank ist die Rentabilität (strukturbruchbereinigt) ${ }^{17}$ mittlerweile deutlich niedriger als in früheren Jahrzehnten. Vor der letzten Finanzkrise mit Tiefpunkt 2009 zeigt sich wie bei den VGR-Zahlen ein auffallender Anstieg bis 2007 und nach 2009 eine Erholung. Dabei wuchs der Personalaufwand

151981 war weltweit Zinsrekordhoch. Der Realzins abzüglich Wirtschaftswachstum stieg um diese Zeit sprunghaft an (Weiß 2014, S. 152). Politisch könnte man die Regierungschefs Margaret Thatcher 1979, Ronald Reagan 1981 und Helmut Kohl 1982 nennen. Ab diesem Zeitpunkt nimmt in den USA auch die Ungleichheit zu (Piketty 2014, S. 44).

16 Der Bereich „unternehmensnahe Dienstleistungen“ ist neu dazu gekommen (vgl. Deutsche Bundesbank 2006, S. 8). Für die letzte Zeitreihe liegt die Rentabilität wieder etwas niedriger, u.a. drücken die jetzt miterfassten Bereiche „Energie" und „Eisenbahnen” die Rentabilität.

17 Soweit die Zeitreihen sich überlappen, können Differenzen ermittelt werden, die konstant fortgeschrieben wurden. 
TABELLE 1

Aufwands- und Ertragsbilanz der Unternehmen

Angaben in Prozent des Gesamtertrags

\begin{tabular}{|c|c|c|c|c|c|c|c|c|c|c|}
\hline & & 1971 & 1980 & 1990 & 1998 & 2004 & 2006 & 2007 & 2009 & 2013 \\
\hline \multirow{3}{*}{ Zinserträge } & $1971-1998$ & 0,5 & 0,5 & 0,6 & 0,6 & & & & & \\
\hline & $1997-2009$ & & & & 0,5 & 0,4 & 0,4 & 0,5 & 0,4 & \\
\hline & $2006-2013$ & & & & & & 0,5 & 0,6 & 0,4 & 0,3 \\
\hline \multirow{3}{*}{ Übrige Erträge } & $1971-1998$ & 2,5 & 2,7 & 3,3 & 4,6 & & & & & \\
\hline & $1997-2009$ & & & & 4,0 & 4,0 & 4,0 & 4,3 & 4,5 & \\
\hline & $2006-2013$ & & & & & & 4,3 & 4,8 & 4,7 & 4,2 \\
\hline \multirow{3}{*}{ Materialaufwand } & $1971-1998$ & 59,1 & 60,9 & 60,3 & 59,4 & & & & & \\
\hline & $1997-2009$ & & & & 58,0 & 59,6 & 61,4 & 61,6 & 60,0 & \\
\hline & $2006-2013$ & & & & & & 61,1 & 60,9 & 61,1 & 63,6 \\
\hline \multirow{3}{*}{ Personalaufwand } & $1971-1998$ & 19,4 & 18,9 & 18,3 & 17,4 & & & & & \\
\hline & $1997-2009$ & & & & 18,5 & 17,3 & 15,7 & 15,4 & 16,6 & \\
\hline & $2006-2013$ & & & & & & 15,4 & 15,0 & 15,6 & 14,8 \\
\hline \multirow{3}{*}{ Abschreibungen } & $1971-1998$ & 3,8 & 3,3 & 3,7 & 3,4 & & & & & \\
\hline & $1997-2009$ & & & & 3,4 & 2,9 & 2,6 & 2,5 & 2,7 & \\
\hline & $2006-2013$ & & & & & & 3,2 & 3,1 & 3,3 & 2,6 \\
\hline \multirow{3}{*}{ Zinsaufwendungen } & $1971-1998$ & 1,6 & 1,7 & 1,5 & 1,2 & & & & & \\
\hline & $1997-2009$ & & & & 1,2 & 1,0 & 0,9 & 1,0 & 1,0 & \\
\hline & $2006-2013$ & & & & & & 1,1 & 1,2 & 1,1 & 1,0 \\
\hline \multirow{3}{*}{ Betriebssteuern } & $1971-1998$ & 2,9 & 2,6 & 2,8 & 3,2 & & & & & \\
\hline & $1997-2009$ & & & & 1,6 & 1,6 & 1,5 & 1,4 & 1,5 & \\
\hline & $2006-2013$ & & & & & & 1,4 & 1,2 & 1,4 & 1,1 \\
\hline \multirow{3}{*}{$\begin{array}{l}\text { Übrige } \\
\text { Aufwendungen }\end{array}$} & $1971-1998$ & 10,0 & 10,4 & 11,4 & 13,4 & & & & & \\
\hline & $1997-2009$ & & & & 13,5 & 13,9 & 13,5 & 13,2 & 14,6 & \\
\hline & $2006-2013$ & & & & & & 13,6 & 13,4 & 14,3 & 13,0 \\
\hline \multirow{3}{*}{$\begin{array}{l}\text { Gesamte } \\
\text { Aufwendungen }\end{array}$} & $1971-1998$ & 96,9 & 97,9 & 97,9 & 98,1 & & & & & \\
\hline & $1997-2009$ & & & & 96,3 & 96,3 & 95,6 & 95,1 & 96,4 & \\
\hline & $2006-2013$ & & & & & & 95,8 & 95,0 & 96,8 & 96,2 \\
\hline \multirow{3}{*}{$\begin{array}{l}\text { Jahresüberschuss } \\
\text { Jahresergebnis }\end{array}$} & $1971-1998$ & 3,1 & 2,1 & 2,1 & 1,9 & & & & & \\
\hline & $1997-2009$ & & & & 2,7 & 2,8 & 3,5 & 3,9 & 2,8 & \\
\hline & $2006-2013$ & & & & & & 3,3 & 4,0 & 2,4 & 3,1 \\
\hline \multirow{3}{*}{$\begin{array}{l}\text { Jahresüberschuss/ } \\
\text { Jahresergebnis vor } \\
\text { Gewinnsteuern }\end{array}$} & $1971-1998$ & - & - & 3,4 & 3,2 & & & & & \\
\hline & $1997-2009$ & & & & 3,7 & 3,7 & 4,4 & 4,9 & 3,6 & \\
\hline & $2006-2013$ & & & & & & 4,2 & 5,0 & 3,2 & 3,8 \\
\hline
\end{tabular}

Quelle: Deutsche Bundesbank; Berechnungen des Autors.

schwächer als der Materialaufwand (Tabelle 1). Auch nach diesen Zahlen stützte also die Lohnkostenentwicklung die Rentabilität.

\subsection{Zeitliche und räumliche Einordnung der Ergebnisse}

Der Rückgang von Kapitalproduktivität und Rentabilität lässt sich für die Bundesrepublik bis in die 1950er Jahre hinein zurückverfolgen. Priewe (1988, S. 142ff., 244f.) gibt für den Zeitraum von 1950 bis 1985 auf der Grundlage von konstanten Preisen Indexreihen für die Kapitalproduktivität und die Profitrate für die Industrie an. ${ }^{18}$ Sie wurden nachrichtlich in die Abbildungen 1 und 2 eingefügt. Für den überlappenden Bereich zwischen 1970 bis 1985 ist der Rückgang allerdings schwächer als bei den in diesem Beitrag berechneten Reihen.

18 Berechnet auf Basis von DIW-Daten (Priewe 1988, S. 142). 
TABELLE 2

\section{Aktivseite der Unternehmensbilanzen}

Angaben in Prozent der Bilanzsumme

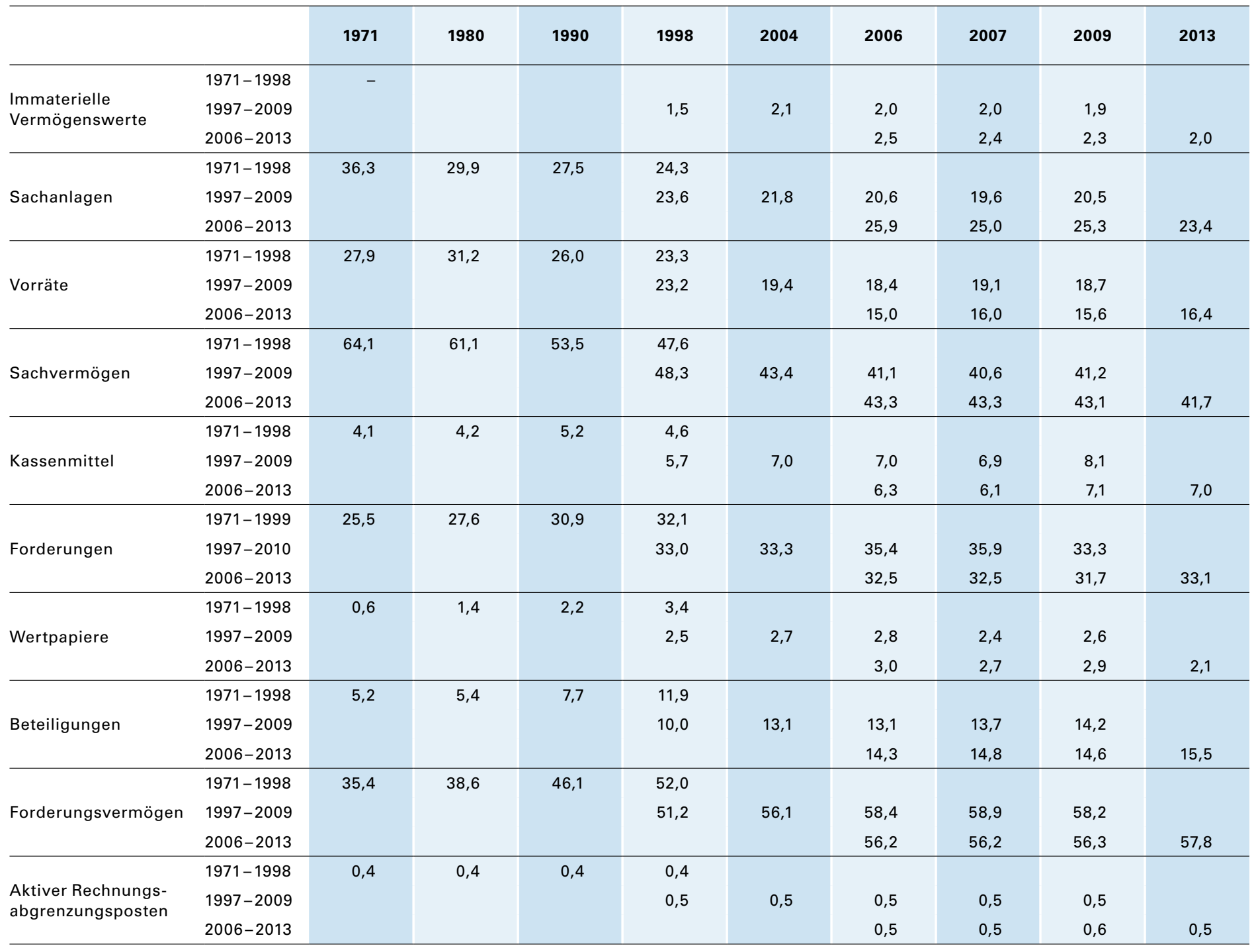

Ipsen (1983, hier nach der Darstellung bei Priewe 1988, S. 239ff.) berechnet für den Unternehmenssektor ohne Wohnungsvermietung Kapitalproduktivität und Rentabilität 1950 bis $1976 .{ }^{19}$ Für den überlappenden Bereich 1970 bis 1976 passt die Reihe für die Kapitalproduktivität gut zur hier vorgestellten Reihe für Wirtschaft ohne Grundstücks- und Wohnungswesen. Bei der Rentabilität verläuft sie günstiger. Tatsächlich war die Lohnentwicklung nach den damaligen Zahlen schwächer als nach den heutigen (hier nicht ausgewiesen).

Nach Lindlar (1997, S. 318) fällt für den Unternehmenssektor ohne Wohnungsbau die Kapitalproduktivität, in konstanten Preisen berechnet, ungefähr linear von knapp $70 \% 1950$ bis $36 \%$ 1982, danach bis zum Ende der
Untersuchungsperiode 1993 abgeschwächt. Auch die Sachkapitalrendite fällt in den dort vorgestellten Untersuchungen für Unternehmenssektor ohne Wohnungsbau und Verarbeitendes Gewerbe seit den 1950er Jahren bis 1982 (ebd., S. 270, 357ff.). Dann kommt es zu einer eher schwach ausgeprägten Erholung bis Anfang der 1990er Jahre. Bontrup (2000) ermittelt auf Grundlage konstanter Preise ebenfalls als Tiefpunkt das Jahr 1982, ab welchem sich die Profitrate bis zum Ende der Untersuchungsperiode 1997 wieder teilweise erholt.

19 Für das Nettoanlagevermögen. 
TABELLE 3

\section{Passivseite der Unternehmensbilanzen}

Angaben in Prozent der Bilanzsumme

\begin{tabular}{|c|c|c|c|c|c|c|c|c|c|c|}
\hline & & 1971 & 1980 & 1990 & 1998 & 2004 & 2006 & 2007 & 2009 & 2013 \\
\hline & $1971-1998$ & 25,3 & 19,7 & 18,2 & 18,7 & & & & & \\
\hline \multirow[t]{2}{*}{ Eigenmittel } & $1997-2009$ & & & & 17,5 & 22,8 & 24,3 & 24,2 & 25,2 & \\
\hline & $2006-2013$ & & & & & & 24,3 & 25,1 & 25,7 & 28,1 \\
\hline \multirow{3}{*}{$\begin{array}{l}\text { Kurzfristige } \\
\text { Verbindlichkeiten }\end{array}$} & $1971-1998$ & 43,2 & 46,7 & 45,5 & 44,5 & & & & & \\
\hline & $1997-2009$ & & & & 45,9 & 43,8 & 43,5 & 44,2 & 42,5 & \\
\hline & $2006-2013$ & & & & & & 40,1 & 40,9 & 39,1 & 40,3 \\
\hline \multirow{3}{*}{$\begin{array}{l}\text { Langfristige } \\
\text { Verbindlichkeiten }\end{array}$} & $1971-1998$ & 20,8 & 18,7 & 15,6 & 14,8 & & & & & \\
\hline & 1997-2009 & & & & 17,5 & 13,5 & 12,4 & 11,4 & 13,1 & \\
\hline & $2006-2013$ & & & & & & 15,7 & 14,7 & 16,0 & 14,7 \\
\hline \multirow{3}{*}{ Rückstellungen } & $1971-1998$ & 10,3 & 14,5 & 20,4 & 21,7 & & & & & \\
\hline & $1997-2009$ & & & & 18,7 & 19,4 & 19,3 & 18,7 & 18,7 & \\
\hline & $2006-2013$ & & & & & & 18,9 & 18,4 & 18,3 & 16,0 \\
\hline \multirow{3}{*}{$\begin{array}{l}\text { Rechnungs- } \\
\text { abgrenzungsposten }\end{array}$} & $1971-1998$ & 0,5 & 0,4 & 0,3 & 0,4 & & & & & \\
\hline & $1997-2009$ & & & & 0,3 & 0,4 & 0,4 & 0,4 & 0,5 & \\
\hline & $2006-2013$ & & & & & & 1,0 & 0,9 & 0,9 & 0,8 \\
\hline \multirow{3}{*}{ Fremdmittel } & $1971-1998$ & 74,2 & 80,0 & 81,5 & 80,9 & & & & & \\
\hline & $1997-2009$ & & & & 82,5 & 77,2 & 75,7 & 74,7 & 74,8 & \\
\hline & $2006-2013$ & & & & & & 75,7 & 74,9 & 74,3 & 71,9 \\
\hline \multirow{3}{*}{ Gesamte Erträge } & $1971-1998$ & 166,9 & 185,8 & 179,2 & 173,5 & & & & & \\
\hline & $1997-2009$ & & & & 188,5 & 184,7 & 190,3 & 186,4 & 170,3 & \\
\hline & $2006-2013$ & & & & & & 165,4 & 162,3 & 151,4 & 159,0 \\
\hline
\end{tabular}

Diese hier vorgestellten Ergebnisse ähneln auch Befunden aus anderen Ländern. Für die USA z. B. markiert ebenfalls ungefähr das Jahr 1982 für die Profitrate einen Tiefpunkt, ab welchem von einer Seitwärtsbewegung gesprochen werden kann. Auch in den USA war im Vorlauf zum Krisenausbruch 2007 ein starker Gewinnanstieg zu beobachten (vgl. Kliman 2012, S. 111; Freeman 2012, S. 171 für das Vereinigte Königreich, S. 179 für die USA). Nach Piketty (2014, S. 259) sinkt seit 1950 die Kapitalproduktivität für die Welt als Ganzes. Für Großbritannien und Frankreich errechnet Piketty ebenfalls ab 1950 sinkende Kapitalrenditen, mit einer vorübergehenden Erholungsphase um die letzte Jahrhundertwende (ebd., S. 268). ${ }^{20}$

\subsection{Der Finanzsektor im Spiegel der Bilanzen}

Die Daten der VGR zeigen, dass der Finanzsektor sich dem allgemeinen Abwärtstrend nicht entzog. Auch hier liegen inzwischen Kapitalproduktivität und Rentabilität langfristig niedriger. Vor dem Krisentiefpunkt 2009 gab es eine „Blase“, der in abgeschwächter Form eine ähnliche Entwicklung in der Industrie folgte. Der Finanzsektor kam auch ein Jahr eher aus dem letzten Krisentief als die Industrie.
Die Daten der Bundesbank geben Hinweise auf den Einfluss des Finanzsektors bei den nichtfinanziellen Unternehmen auf Aktiv- und Passivseite der Bilanz und bei Aufwand und Ertrag. So nahmen auf der Aktivseite die Kassenmittel seit 1970 von $4 \%$ der Bilanzsumme auf zuletzt $7 \%$ zu. Zu erwartende Einsparungen infolge von Rationalisierungen sind nicht erkennbar und somit bleibt die Rentabilität durch Kassenhaltung belastet (Tabelle 2). Dies deutet auf Unsicherheit als Motiv für Kassenhaltung („Kriegskassen“, vgl. Bernanke 2005).

Auf der Aktivseite gewinnt die Finanzsphäre an Bedeutung, weil der Anteil des Forderungsvermögens an der Bilanzsumme zulasten des Sachvermögens von 1970 knapp $40 \%$ auf zuletzt knapp $60 \%$ ungefähr linear zugenommen hat (Tabelle 2). Dies ist der Trend zur „Bank mit angeschlossener Werkstatt“. Als Gegenbuchungen kommen - über die Banken vermittelt - eine zunehmende Verschuldung des Staates, der privaten Haushalte oder des Auslands, etwa

20 „Kapital“ wie bei Statistisches Bundesamt/Deutsche Bundesbank (2013) definiert. 
in Form von Beteiligungen an ausländischen Unternehmen, infrage. So haben Beteiligungen von 5 auf $15 \%$ zugenommen. Dieses Umlenken von Anlagen in Finanzvermögen geht zulasten der realen Kapitalakkumulation (zu Letzterer vgl. Knetsch 2013; siehe auch Weiß 1998). Die Unternehmen finanzierten so das „Exportmodell Deutschland“ und die „Binnenexporte“ (vgl. Kalecki 1987) an den Staat. Die Passivseite der Unternehmensbilanzen (Tabelle 3) schließlich zeigt ein Verhältnis von Eigenmitteln zu Fremdmitteln von etwa 1:3, vorübergehend um das Jahr 2000 von $1: 4$.

\section{Zusammenfassung und Bewertung}

Die Untersuchung hat für die VGR-Zahlen gezeigt, dass für die Gesamtwirtschaft, das Produzierende Gewerbe sowie das Kredit- und Versicherungsgewerbe Kapitalproduktivität und Rentabilität sich langfristig zum Teil deutlich vermindert haben. Im Kredit- und Versicherungswesen lagen die Kapitalproduktivität und die Rentabilität höher als in der Gesamtwirtschaft und im Produzierenden Gewerbe. Die Schwankungsbreite war aber gleichzeitig ebenfalls höher. Im Grundstücks- und Wohnungswesen lagen die Kapitalproduktivität und die Rentabilität weit unter den Werten der anderen Bereiche. Sie wiesen aber eine ungleich stabilere Entwicklung auf. Ein Abwärtstrend ist hier nicht zu beobachten. Seit 1982 ist eine deutliche Verlangsamung der Entwicklung festzustellen. Auch die Zahlen zu den Jahresabschlüssen der Unternehmen zeigen eine Verminderung der Rentabilität. Eine Stabilisierung der Entwicklung lässt sich hier etwa Ende der 1990er Jahre ausmachen.

Der Rückgang und das anhaltend niedrige Niveau der Rentabilität und mehr noch der Kapitalproduktivität in wichtigen Sektoren der Volkswirtschaft sind für die Wirtschafts- und Sozialpolitik von erheblicher Bedeutung. Grundsätzlich kann die Wirtschaftspolitik von der Nachfrage- oder der Angebotsseite eingreifen. Mehr Nachfrage führt zu höherer Kapazitätsauslastung und so zu höherer Kapitalproduktivität (Pettis 2013, S. 6, S. 52ff., S. 79f.; Bontrup 2000, S. 725; Mattfeldt 2006, S. 11). Carl Christian von Weizsäcker (2012) schlägt z. B. mehr staatliche Nachfrage vor. In seiner Deutung der Langfristentwicklung ist inzwischen ein Punkt erreicht, in dem ein Zinssatz sogar von Null noch zu hoch ist, um für die private Ersparnis eine ausreichende Menge an Investitionen auszulösen. Finanziert der Staat nicht mit Staatsverschuldung mehr staatliche Nachfrage und bindet so einen Teil der privaten Ersparnis, drohen eine "Sparschwemme“ und eine wirtschaftliche Abwärtsspirale. Von Weizsäcker knüpft damit an Ben Bernanke (2005) an. Dieser sieht mit seiner These von der „Sparschwemme" (savings glut) die steigende Kapitalintensität (capital-labor ratio) als Ursache für einen Anlagenotstand (dearth of investment opportunities) mit niedrigen Rentabilitätsaussichten. ${ }^{21}$ Nicht zuletzt deswegen verteidigte der ehemalige US-Notenbankchef seine , außergewöhnlichen Maßnahmen“ der Geldpolitik. So ist geldpolitisch angesichts der niedrigen

21 „With slowly growing or declining workforces, as well as high capital-labo ratios, many advanced economies outside the United States also face an apparent dearth of domestic investment opportunities." Ähnlich Pettis $(2013$, S. 1): "overly abundant capital and low interest rates".

22 Vgl. Funke (1987) und die dort angegebene Literatur zur Kontroverse zwischen DIW und RWI in den 1980er Jahren, siehe auch Mattfeldt (2006), S. 11.
Rentabilitäten auch für Deutschland eine „Niedrigzinspolitik“ der Zentralbank eine notwendige und keine freie geldpolitische Entscheidung.

Die Arbeitgeberseite fordert angesichts einer sinkenden Rentabilität hingegen - wenigstens traditionell - statt Nachfragepolitik Maßnahmen auf der Angebotsseite, etwa verteilungspolitische Zugeständnisse. ${ }^{22}$ Doch die sinkende Lohnquote stützte zwar die Rentabilität etwa ab den 1980er Jahren von der Kostenseite her, aber angesichts einer rückläufigen Kapitalproduktivität eben nicht nachhaltig. Dazu kommt, dass die Investitionstätigkeit trotz möglicher Stabilisierung der - durchschnittlichen - Sachkapitalrendite auch in Deutschland nicht in Gang kam (Priewe/Rietzler 2010).

Insgesamt konnten weder geldpolitische Expansion noch lohnpolitische Zurückhaltung nachhaltig Kapitalproduktivität und Rentabilität anheben. Wenn es richtig ist, dass privatwirtschaftliche Investitionen über eine Art „Rationalitätenfall“ “ $z$ war einzelwirtschaftlich die Konkurrenzfähigkeit stärken, gesamtwirtschaftlich aber die Rentabilität senken und so Stagnation fördern („Marx-Effekt“), dann bedarf es staatlicher Eingriffe für Investitionen. Es reicht nicht, allein auf Privatinitiative oder Konkurrenzfähigkeit zu setzen. Es sollten auch in „unproduktiven“, d. h. nicht zu mehr Gewinnen führenden Bereichen sozial sinnvolle Arbeitsplätze geschaffen werden. Industrie- und strukturpolitische staatliche Maßnahmen sowie die staatliche Förderung von Beschäftigung in marktwirtschaftlich nicht rentablen Bereichen werden notwendig (vgl. zu einzelnen Vorschlägen z. B. DGB-Bundesvorstand 2012). Sie vermeiden den einzelwirtschaftlich, aber eben nicht gesamtwirtschaftlich sinnvollen Überaufbau von Kapazitäten. Eine Antwort auf den Einwand, der Staat sei nicht der „bessere Investor“, ist demokratisch zivilgesellschaftliche Beteiligung.

\section{LITERATUR}

Arbeitskreis „Volkswirtschaftliche Gesamtrechnungen der Länder“ (2014): Bruttoinlandsprodukt, Bruttowertschöpfung in den Ländern der Bundesrepublik Deutschland 1991 bis 2013, Reihe 1, Band 1, Frankfurt a. M. Arnold, L. (1997): Wachstumstheorie, München

Bernanke, B. (2005): The global saving glut and the U.S. current account deficit, remarks at the Sandridge Lecture, Virginia Association of Economists, Richmond, Virginia, March 10

Bischoff, J. et al.(1993): Ausbeutung, Selbstverrätselung, Regulation - Der 3. Band des „Kapital", Hamburg

Bitz, M./Schneeloch, D./Wittstock, W. (2011): Der Jahresabschluss, München Bontrup, H. (2000): Zur säkularen Entwicklung der Kapitalrentabilität, WSI-Mitteilungen 53 (11), S. 718-725

Callinicos, A. (2014): Deciphering capital, Marx's capital and its destiny, London

Deutsche Bundesbank (1999): Statistische Sonderveröffentlichung 5, Jahresabschlüsse westdeutscher Unternehmen 1971 bis 1996, Frankfurt a. M. Deutsche Bundesbank (2005): Ertragslage und Finanzierungsverhältnisse deutscher Unternehmen - eine Untersuchung auf neuer Datenbasis, in: Monatsbericht der Deutschen Bundesbank 57 (10), S. 33-54, Frankfurt a. M. Deutsche Bundesbank (2006): Statistische Sonderveröffentlichung 5, Hochgerechnete Angaben aus Jahresabschlüssen deutscher Unternehmen von 1994 bis 2003, Frankfurt a. M.

Deutsche Bundesbank (2009): Statistische Sonderveröffentlichung 5, Hochgerechnete Angaben aus Jahresabschlüssen deutscher Unternehmen von 1997 bis 2007 , Frankfurt a. M.

Deutsche Bundesbank (2013): Statistische Sonderveröffentlichung 6, Verhältniszahlen aus Jahresabschlüssen deutscher Unternehmen von 2009 bis 2010, Frankfurt a. M.

Deutsche Bundesbank (2014a): Säkulare Stagnation und Wirtschaftswachstum, in: Monatsbericht 66 (4), S. 15-18

Deutsche Bundesbank (2014b): Hochgerechnete Angaben aus Jahresabschlüssen 2006 bis 2013, Erläuterungen, Frankfurt a. M. 
DGB-Bundesvorstand (2012): Ein Marshallplan für Europa, Berlin

Freeman, A. (2012): The profit rate in the presence of financial markets: A necessary correction, in: Journal of Australian Political Economy 70 (Summer 2012/2013), S. 167-192

Fröhlich, N. (2010): Die Überprüfung klassischer Preistheorien mithilfe von Input-Output-Tabellen, in: Wirtschaft und Statistik 2010 (5), S. 503-508 Funke, M. (1987): Einflüsse auf die Entwicklung der Kapitalrentabilität im verarbeitenden Gewerbe in der Bundesrepublik Deutschland, in: Jahrbuch für Sozialwissenschaft 38 (2), S. 188-209

Heinrich, M. (2004): Kritik der politischen Ökonomie - eine Einführung, Stuttgart Internationaler Währungsfond (IWF) (2005): World economic outlook September

Ipsen, D. (1983): Die Stabilität des Wachstums, Frankfurt a. M.

Kalecki, M. (1987): Krise und Prosperität im Kapitalismus: Ausgewählte Essays 1933-1971, Marburg

Keynes, J. M. (2009): Allgemeine Theorie der Beschäftigung, des Zinses und des Geldes, 11. erneut verbesserte Auflage, Berlin

KIär, E. (2013): Kapitalakkumulation, Gesamtnachfrage, Arbeitsmarktinstitutionen und Beschäftigung in pfadabhängigen Volkswirtschaften. Neue neoklassische Synthese und postkeynesianische Kritik, Marburg

KIär, E. (2014): Die Eurokrise im Spiegel der Potentialschätzungen - Lehren für eine alternative Wirtschaftspolitik?, Wiso-Diskurs, April 2014, Friedrich-EbertStiftung, Berlin

Kliman, A. (2012): The failure of capitalist production - underlying causes of the Great Recession, London

Knetsch, T. A. (2013): Ein nutzungskostenbasierter Ansatz zur Messung des Faktors Kapital in aggregierten Produktionsfunktionen, in: Jahrbücher für Nationalökonomie und Statistik $233(5+6)$, S. 638-660

Krüger, S. (2012): Keynes \& Marx - Darstellung und Kritik der "GeneralTheory" Bewertung keynesianischer Wirtschaftspolitik - Linker Keynesianismus und Sozialismus, Kritik der Politischen Ökonomie und Kapitalismusanalyse Band 4 Hamburg

Krugman, P. (2013): Secular stagnation, coalmines, bubbles, and Larry Summers, http://krugman.blogs.nytimes.com/2013/11/16/secular-stagnationcoalmines-bubbles-and-larry-summers/?_php=true\&_type=blogs\&_r=0 Lindlar, L. (1997): Das mißverstandene Wirtschaftswunder. Schriften zur angewandten Wirtschaftsforschung 77, Tübingen

Marx, K./Engels, F. (1987): Werke, Band 25, „Das Kapital“, Band III, Berlin Mattfeldt, H. (2006): Zur Methode der Profitratenbestimmung. Anmerkungen zur Empirie der "säkularen Entwicklung der Kapitalrentabilität", ZÖSS Discussion Paper (09), Universität Hamburg

Nachtwey, O. (2015): Die Abstiegsgesellschaft - Über das Aufbegehren in der regressiven Moderne, Berlin (im Erscheinen)

Pettis, M. (2013): The great rebalancing. Trade, conflict, and the perilous road ahead for the world economy, Princeton

Piketty, T. (2014): Das Kapital im 21. Jahrhundert, München
Priewe, J. (1988): Krisenzyklen und Stagnationstendenzen in der Bundesrepublik Deutschland: Die krisentheoretische Debatte, Köln

Priewe, J./Rietzler, K. (2010): Deutschlands nachlassende Investitionsdynamik 1991 - 2010. Ansatzpunkte für ein neues Wachstumsmodell: Friedrich Ebert Stiftung, Abteilung Wirtschafts- und Sozialpolitik, Wiso-Diskurs,

Expertisen und Dokumentationen zur Wirtschafts- und Sozialpolitik, Berlin Räth, N. et al. (2011): Revision der Volkswirtschaftlichen Gesamtrechnung 2011 für den Zeitraum 1991 bis 2010, in: Wirtschaft und Statistik 2011 (9), S. 825-865 Schmalwasser, O./Schidlowski, M. (2006): Kapitalstockrechnung in Deutschland, in: Wirtschaft und Statistik 11/2006, S. 1107-1123

Statistisches Bundesamt (2013), Fachserie 18, Volkswirtschaftliche Gesamtrechnungen, Inlandsproduktsberechnung, Detaillierte Jahresergebnisse, Wiesbaden

Statistisches Bundesamt (2014a): Volkswirtschaftliche Gesamtrechnungen, Bruttoinlandsprodukt, Bruttonationaleinkommen, Volkseinkommen, Lange Reihen ab 1925, Wiesbaden

Statistisches Bundesamt (2014b): VGR-Generalrevision 2014, Ergebnisse und Hintergründe, Hintergrundpapier zur Pressemitteilung vom 01. September, Wiesbaden

Statistisches Bundesamt/Deutsche Bundesbank (2013): Sektorale und gesamtwirtschaftliche Vermögensbilanzen 1991-2012, Wiesbaden Summers, L. (2013): Larry Summers remarks, IMF Annual Research Conference, November 8th

Weiß, T. (1998): „Turbokapitalismus“? - Zu derzeitigen weltwirtschaftlichen Problemen, in: WSI-Mitteilungen 51 (12), S. 837-844

Weiß,T. (2014): Arbeitsproduktivität, nicht nur eine zentrale arbeitsökonomische und makroökonomische Kategorie, in: Laske, S./Schweres M. (Hrsg.): Arbeitsorientierung in den Wirtschaftswissenschaften, Schriftenreihe zur interdisziplinären Arbeitswissenschaft Band 2, München, S.137-155 Weizsäcker, C. C. von (2012): Grenzen des Konzepts einer unabhängigen Zentralbank, in: Wirtschaftsdienst 92 (2), S. 91-94

\section{AUTOR}

THOMAS WEIß, Dr. rer.pol, ist Referent im Bundesministerium für Arbeit und Soziales. Arbeitsschwerpunkte: Konjunktur-, Struktur- und Wachstumsanalysen, Verteilungsfragen, Sozialversicherung in den Volkswirtschaftlichen Gesamtrechnungen.

thomas.weiss@bmas.bund.de 